\title{
A regenerative microchannel device for recording multiple single-unit action potentials in awake, ambulatory animals
}

\author{
Akhil Srinivasan, ${ }^{1}$ John Tipton, ${ }^{1}$ Mayank Tahilramani, ${ }^{1}$ Adel Kharbouch, ${ }^{1}$ Eric Gaupp, ${ }^{1}$ Chao Song,${ }^{2}$ \\ Poornima Venkataraman, ${ }_{1}$ Jessica Falcone, ${ }^{1}$ Stéphanie P. Lacour, ${ }^{3}$ Garrett B. Stanley, ${ }^{1}$ Arthur W. English ${ }^{4}$ \\ and Ravi V. Bellamkonda ${ }^{1}$ \\ ${ }^{1}$ Wallace H. Coulter Department of Biomedical Engineering, Georgia Institute of Technology and Emory University School of \\ Medicine, 313 Ferst Drive, Atlanta, GA 30332, USA \\ ${ }^{2}$ School of Electrical and Computer Engineering, Georgia Institute of Technology and Emory University School of Medicine, \\ Atlanta, GA, USA \\ ${ }^{3}$ Centre for Neuroprosthetics, School of Engineering, Institute of Microengineering and Institute of Bioengineering, Ecole \\ Polytechnique Fédérale de Lausanne, Switzerland \\ ${ }^{4}$ Department of Cell Biology, Emory University School of Medicine, Atlanta, GA, USA
}

Keywords: nerve conduction, nerve injury and regeneration, neural interfacing, neural recordings, regenerative neural interface

Edited by Paul Bolam

Received 5 June 2015, revised 7 August 2015, accepted 8 September 2015

\begin{abstract}
Despite significant advances in robotics, commercially advanced prosthetics provide only a small fraction of the functionality of the amputated limb that they are meant to replace. Peripheral nerve interfacing could provide a rich controlling link between the body and these advanced prosthetics in order to increase their overall utility. Here, we report on the development of a fully integrated regenerative microchannel interface with 30 microelectrodes and signal extraction capabilities enabling evaluation in an awake and ambulatory rat animal model. In vitro functional testing validated the capability of the microelectrodes to record neural signals similar in size and nature to those that occur in vivo. In vitro dorsal root ganglia cultures revealed striking cytocompatibility of the microchannel interface. Finally, in vivo, the microchannel interface was successfully used to record a multitude of single-unit action potentials through $63 \%$ of the integrated microelectrodes at the early time point of 3 weeks. This marks a significant advance in microchannel interfacing, demonstrating the capability of microchannels to be used for peripheral nerve interfacing.
\end{abstract}

\section{Introduction}

Today, neural prosthetics provide a small fraction of the functionality of the natural amputated limb that they are meant to replace. Advances in robotics have led to high-dimensionality prosthetic robotic limbs, but the control interface remains the 'weak link', largely because of the lack of reliable interfacing with the nervous system. Peripheral nerve interfaces (PNIs) constitute a logical control source for limb prosthetics, and can potentially enable specific engagement of large numbers of independent electrodes with the peripheral nerve. However, penetrating PNIs, such as the Utah Slanted Electrode Array, elicit chronic inflammation and scar formation (Branner \& Normann, 2000; Branner et al., 2001, 2004; Navarro et al., 2005). Cuff electrodes have seen clinical application and circumvent this injury to the nerve, but do so at the expense of recording and stimulation specificity (Fisher et al., 2008, 2009; Ortiz-Catalan et al., 2014). The cuff electrodes reside outside the nerve epineurium, where the distance from axons is too great for

Correspondence: R. V. Bellamkonda, as above

E-mail: ravi@gatech.edu, bellamkonda@emory.edu recording of single-unit action potentials (APs) (Navarro et al., 2005; Fitzgerald et al., 2008). Accordingly, although they are helpful in a number of clinical applications, their overall utility is potentially limited. Regenerative PNIs, including the traditional sieve electrode, theoretically provide a balance of specificity without additional nerve injury after amputation by allowing nerves and axons to regenerate close to integrated electrodes (Negredo et al., 2004; Lago et al., 2005, 2007; Garde et al., 2009; Clements et al., 2013). This approach necessitates a target clinical population of amputees or individuals with a nerve injury where the device can be implanted at the time of surgery. Unfortunately, all of these PNIs fail to record large numbers of single-unit APs from axons chronically, and are unable to realize the full functionality of a robotic limb (Rutten, 2002; Branner et al., 2004; Navarro et al., 2005; Yoo \& Durand, 2005; Ramachandran et al., 2006; Lago et al., 2007; Fitzgerald et al., 2008).

Microchannel-based peripheral nerve interfacing is a relatively new approach to peripheral nerve interfacing, and offers significant advantages over previous approaches. One of the fundamental challenges with peripheral nerve interfacing is the low-impedance extracellular fluid surrounding axons. This low-impedance fluid 
significantly reduces the extracellular amplitude of APs by effectively shunting them to ground, and as a result reduces the viability of all previous PNI approaches (Fitzgerald et al., 2008). Another important consideration is the node of Ranvier placement, i.e. locations along an axon where the AP is largest, which occur at least every 2 mm (Hess \& Young, 1952; Salzer, 1997). This forces a spatial requirement on peripheral nerve interfacing, where electrodes need to be as close to the nodes as possible. Previous PNI approaches, such as the sieve electrodes, are unable to take this spatial requirement into account, again reducing overall electrode viability (Rutten, 2002; Navarro et al., 2005; Lago et al., 2007; Fitzgerald et al., 2008). In contrast, axons can be placed or guided to regenerate through small microchannels of the order of $100 \mu \mathrm{m}$ in diameter. Doing so effectively constrains the low-impedance extracellular fluid, thereby increasing the extracellular resistance and preventing the AP signal from dissipating. As a result, the extracellular amplitudes of the APs are significantly larger, enabling higher signal-to-noise recordings (Fitzgerald et al., 2008). Furthermore, making the microchannels longer than $2 \mathrm{~mm}$ effectively guarantees the presence of a node of Ranvier of each axon within the microchannel. This, coupled with the increased extracellular resistance, enables recordings that are independent of the node of Ranvier position, because the amplitude of the AP signal is relatively constant throughout the microchannel (Fitzgerald et al., 2008). This theory has been modelled and well validated in previous in vitro studies (Fitzgerald et al., 2008; FitzGerald et al., 2009).

Previous in vivo studies have focused on evaluating the efficacy of recording single-unit APs by using electrodes contained within the microchannels. These rodent studies have ranged from acute recordings, where axons have been placed within microchannels, to recordings after chronic implantation, where axons have regenerated through microchannels (Delivopoulos et al., 2012; FitzGerald et al., 2012; Minev et al., 2012; Chew et al., 2013; Gore et al., 2015; Srinivasan et al., 2015). Significant work has also been performed on validating the chronic stability of nerves and axons after they have regenerated into and through microchannels, with an eye towards clinical translation, including an amputee population (Yao et al., 2010; Tansey et al., 2011; Srinivasan et al., 2015). However, none of these previous studies has evaluated a fully integrated microchannel interface with a large number of microelectrodes and signal extraction capabilities enabling recordings in awake and freely moving rodents.

Here, we have developed and evaluated a microchannel interface, the Georgia Tech Regenerative Electrode (GT-RE), and its ability to be utilized for challenging single-unit AP recordings in awake and freely moving rodents. First, the in vitro functionality of the GT-RE was validated by the use of artificial neural signals. Second, in vitro dorsal root ganglia (DRG) studies were utilized to validate cytocompatibility after microfabrication of the GT-RE, which involved a number of toxic solvents and processes. Finally, in vivo, the large electrode count GT-RE was implanted in the rat sciatic nerve transection model. In vivo recordings were performed while rats were awake and ambulating, to evaluate the capability to record singleunit APs through microchannel interfaces.

\section{Materials and methods}

\section{GT-RE design}

The basic concept of the GT-RE microchannel interface is shown in Fig. 1. The implant was formed by rolling a planar (two-dimensional) array of parallel microchannels into a structure matching the nerve diameter. A microelectrode array (MEA) was embedded into a flexible

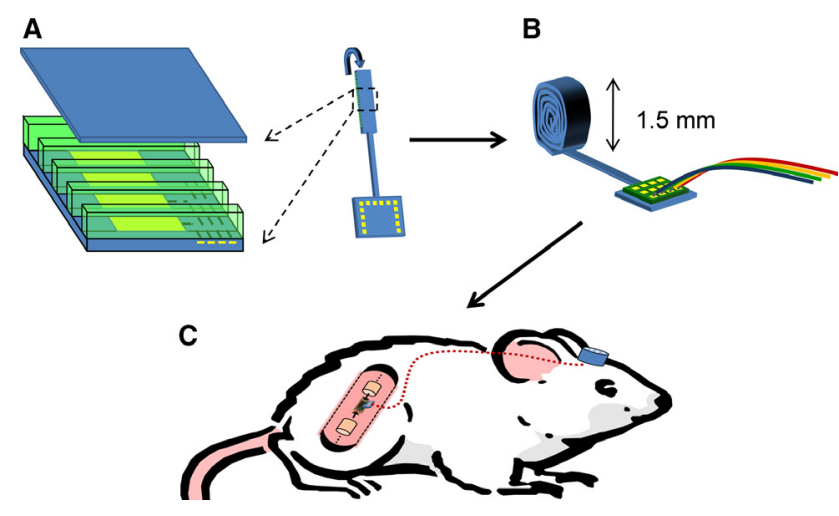

FIG. 1. Schematic of a fully integrated microchannel interface, the GT-RE. (A) Microchannel architecture showing a blue polymer substrate with integrated gold microelectrodes and traces leading to bonding pads, green SU-8 microchannel walls, and a blue top capping polymer layer forming a sheet of microchannels with integrated electrodes. (B) Rolling of the microchannels to form a cylinder of microchannels and integration of a surface mount PCB with signal extraction wires. (C) Implantation of the GT-RE in the rat sciatic nerve model, in which signal extraction wires are burrowed subcutaneously to a percutaneous headcap mounted on the skull to enable neural signal recordings.

Parylene C layer. The electrodes were $80 \mu \mathrm{m} \times 40 \mu \mathrm{m}$ rectangles with the long axis oriented along the width of the microchannels and the short axis oriented along the length. The electrodes were spaced once every third microchannel to allow for an equal distribution of electrodes throughout the cross-section of the GT-RE post-rolling. A common bipolar reference was placed near the bonding pads, and a common pseudo-tripolar reference was designed with electrode sites at the entrance and exit of every microchannel containing an electrode. In some cases during in vivo testing, a local low-impedance wire was used as a bipolar reference instead. The referencing design and the electrodes, traces and bonding pads are shown in Fig. 2.

SU-8 microchannel walls were patterned on top of the flexible MEA, and a capping polydimethylsiloxane (PDMS) layer was then placed on top of the SU-8 microchannel walls. This formed a sheet of 97 microchannels with electrode sites housed in the base of the microchannels. This sheet was rolled perpendicularly to the direction of the microchannels, forming a cylinder matching the shape and size of the rat sciatic nerve. The microchannels were $100 \mu \mathrm{m}$ in width, $100 \mu \mathrm{m}$ in height, and $3.5 \mathrm{~mm}$ in length, with $20-\mu \mathrm{m}$-wide microchannel walls. These dimensions were based on previous work that, in part, investigated microchannel sizes and their relationship with nerve regeneration in a clinically translatable amputee animal model (Srinivasan et al., 2015).

Metal traces connected to the microelectrodes in the microchannels ended at a series of bonding pads intended for surface mount connection to a printed circuit board (PCB). The PCB integrated with the GT-RE was used as a wire attachment hub, and had 34 multistranded stainless steel wires (0.001-inch Stainless Steel 7 strand PFA; A-M Systems) soldered accounting for the electrodes, references, and a local ground. These signal extraction wires were connected to an Omnetics 36 channel percutaneous connector, which allowed eventual connection to a separate off-board amplifier for in vivo electrophysiology experimentation.

\section{GT-RE fabrication}

\section{Parylene C MEA fabrication}

To fabricate the flexible Parylene C MEA, a $10-\mu \mathrm{m}$ base layer of Parylene $\mathrm{C}$ was first deposited on glass coated with $2 \%$ Micro-Clean 

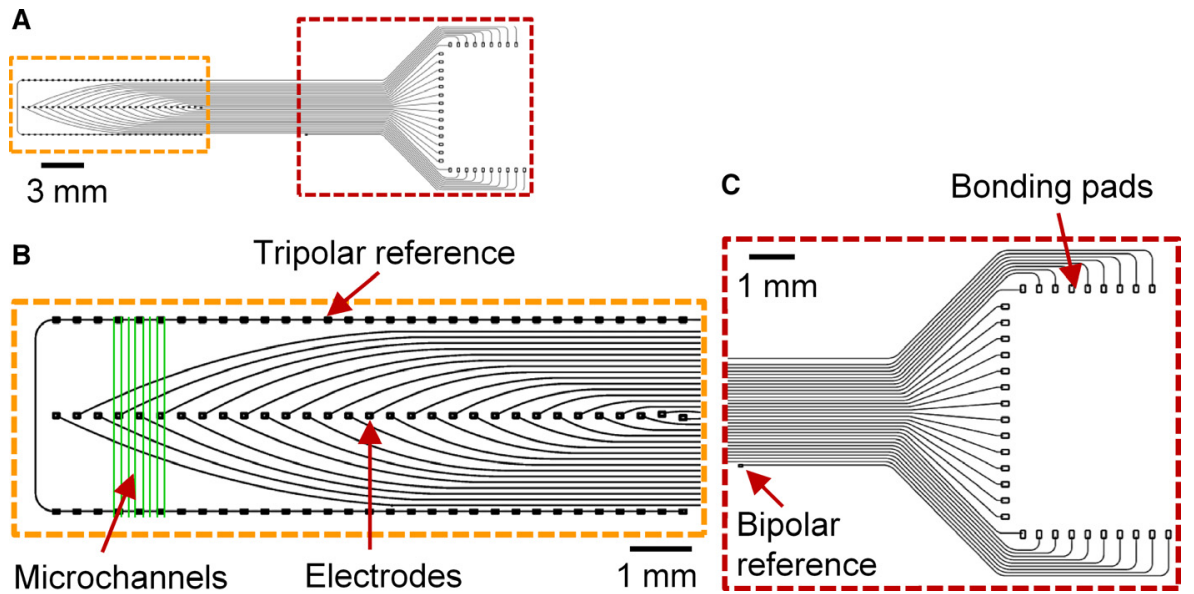

FIG. 2. Design of GT-RE electrodes, references, traces, and bonding pads. (A) Overall design. (B) Close up of the electrode region and tripolar reference. Example SU-8 microchannel walls are also depicted in green. (C) Close up of the bonding pads and bipolar reference.

solution to allow easy removal post-fabrication. This was followed by lift-off patterning to define the titanium/gold $(100 \AA \mathrm{Ti} / 1000 \AA$ $\mathrm{Au})$ electrodes, traces/interconnects, and bonding pads. A negative photoresist, NR4-8000P, was spun, baked, and exposed to an experimentally determined ultraviolet exposure dose of $590 \mathrm{~mJ} / \mathrm{cm}^{2}$. The sample was then post-baked and developed to produce a lift-off mask for metal deposition. Descumming with $\mathrm{O}_{2}$ plasma was performed from $30 \mathrm{~s}$ to $3 \mathrm{~min}$ to further clear out photoresist residues. Sputtering of the thin metal film bi-layers through the mask followed after activation of the surface of the exposed Parylene $\mathrm{C}$ substrate with brief $\mathrm{O}_{2}$ plasma (power, $70 \mathrm{~W}$; pressure, $100 \mathrm{mTorr}$; duration, $60 \mathrm{~s}$ ) to ensure reliable adhesion of the metal film to the polymer. The photoresist was then stripped, leaving the patterned MEA. These fabrication steps are shown in Fig. 3A, with the basic steps numbered.

After photoresist stripping and an additional $\mathrm{O}_{2}$ plasma activation step, the MEA was encapsulated with a 5- $\mu$ m-thick film of Parylene C. This was followed by patterning an etching mask for the Parylene $\mathrm{C}$ passivation layer used during etching of the passivation to expose the microelectrodes and bonding pads. The photoresist SPR 220-7.0 was spun, baked, and exposed to an experimentally determined exposure dose of $150 \mathrm{~mJ} / \mathrm{cm}^{2}$. The sample was then developed and hard-baked to anneal the remaining SPR 220-7.0 forming the etch mask, where only the Parylene $\mathrm{C}$ passivation layer covering the electrodes and bonding pads was exposed for etching. Etching of the Parylene $\mathrm{C}$ passivation layer was performed with $\mathrm{O}_{2}$ plasma, and the gold electrodes and bonding pads served as an etch stop layer. After the etching, the remaining photoresist was stripped. Two of the devices tested in vivo utilized $15-\mu \mathrm{m}$-thick and $10-\mu \mathrm{m}$ thick Parylene $\mathrm{C}$ base and passivation layers, respectively, to aid in physical handling and Parylene $\mathrm{C}$ layer adhesion. These fabrication steps are shown in Fig. 3B, with the basic steps numbered.

\section{Microchannel fabrication}

First, microchannel walls were patterned onto the Parylene C MEAs. The basic process of patterning SU-8 microchannel walls has been reported previously (Srinivasan et al., 2015). Briefly, a $100-\mu \mathrm{m}$ layer of SU-8 was spun on the MEAs. After spinning, the MEAs were subjected to pre-exposure baking, exposure, post-exposure
A 1

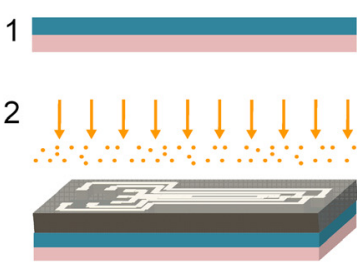

3

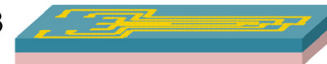

B 1
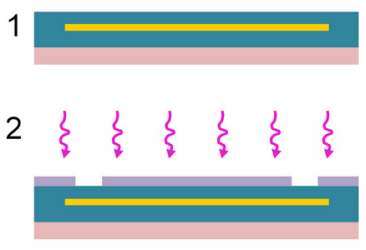

3

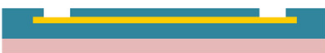

C

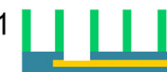

2

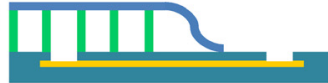

Glass Parylene C

Photoresist Metal

Photoresist $\left\{\mathrm{O}_{2}\right.$ plasma

SU-8 PDMS

FIG. 3. GT-RE fabrication schematic. (A) Metal patterning by use of a liftoff approach to define electrodes, traces, and bonding pads. (B) Patterning of the passivation layer by use of an etching mask. (C) Addition of the SU-8 microchannel walls and PDMS capping layer forming the microchannels. 
baking, and finally development, resulting in microchannel walls being patterned on top of the MEAs. In this case, the MEAs were not pretreated with $\mathrm{O}_{2}$ plasma as reported previously, owing to incomplete development of SU-8 after $\mathrm{O}_{2}$ plasma treatment.

After the microchannel wall fabrication, the capping PDMS layer was applied. First, a 40- $\mu$ m PDMS layer was spun on clean glass, forming the 'top PDMS substrate'. This was a permanent PDMS layer, and not part of the GT-RE. Its purpose was to account for slight variability in SU-8 microchannel wall heights, by ensuring contact between the PDMS cover layer and the entire surface area of every microchannel wall. This was then briefly treated with $\mathrm{O}_{2}$ plasma to reduce PDMS hydrophobicity and enable uniform spin-coating of a polyacrylic acid film, a water-resorbable layer. After drying on a hotplate at $65{ }^{\circ} \mathrm{C}$ for $5 \mathrm{~min}$, a 5- $\mu \mathrm{m}$ PDMS layer was spun on and cured by use of a hotplate at $90{ }^{\circ} \mathrm{C}$ for $20 \mathrm{~min}$, without allowing the polyacrylic acid to cool or absorb ambient moisture. After curing, the top PDMS substrate was briefly subjected to $\mathrm{O}_{2}$ plasma again, to aid adhesion, and a second 5- $\mu \mathrm{m}$ PDMS layer was immediately spun on. During this time, the MEA with SU-8 microchannel walls facing upwards was placed on a hotplate set to $90{ }^{\circ} \mathrm{C}$ in preparation for the top PDMS substrate. Immediately after spinning of the second 5- $\mu$ m PDMS layer, the top PDMS substrate was immediately placed on the MEA substrate under weight, with the uncured 5- $\mu \mathrm{m}$ PDMS layer and SU-8 microchannel walls in contact with each other. The two substrates (MEA and top PDMS layer) were cured together on the hotplate at $90{ }^{\circ} \mathrm{C}$ for $20 \mathrm{~min}$. After cooling, the substrates were soaked in water until the polyacrylic acid dissolved and the top PDMS glass substrate slid easily offm leaving the capping $10-\mu \mathrm{m}$ PDMS layer securely adhered to the SU-8 microchannel walls. The capping 10- $\mu \mathrm{m}$ PDMS layer was formed from the two 5- $\mu$ m PDMS layers spun on top of each other. The addition of the SU-8 microchannel walls and PDMS capping layer is shown in Fig. 3C.

Finally, the MEAs with complete microchannels were peeled off the glass substrate, and the microchannel region was rolled. Importantly, the rolling took place along the Parylene C substrate layer, forcing the capping PDMS layer to stretch. If the sheet were to be rolled around the top PDMS layer, it would buckle and block the microchannels. To aid in the rolling process, silicone adhesive was manually applied to the midline of the microchannel region on the back of the Parylene $\mathrm{C}$ substrate layer. The silicone adhesive was used to attach each consecutively rolled layer to the previous layer to prevent unrolling.

\section{Signal extraction wires and hermetic packaging}

Bonding pads on the GT-RE were designed to match those on a PCB wire hub to be connected with a surface mount approach and silver-based conductive epoxy (H20E; Epoxy Technology) as the junction between the two sets of bonding pads. Thirty-four wires coming off the PCB were divided into separate groups of 17 wires, and a three-braid bundle was utilized with sets of three, four and four wires for mechanical reinforcement and to prevent breakage in vivo. Each braid was fed into a silicone tube to act as an extra layer of insulation and mechanical protection.

Molds for insulating and encapsulating the $\mathrm{PCB}$ region (PCB mould) and headcap (headcap mould) of the GT-REs were first three-dimensionally printed by use of an Objet Eden 250 (Stratasys). A uniform 10- $\mu$ m layer of the AZ P4620 photoresist (MicroChemicals) was then spray-coated on all surfaces of the moulds with a Suss MicroTec AltaSpray coater, baked, and exposed. The photoresist served as an eventual dissolvable release layer to release the GT-RE PCB region and headcap from the moulds.
Silicone adhesive (40076; Applied Silicone Corporation) was applied to the cable region between the microchannels and the bonding pads for mechanical protection during handling. The silicone adhesive was applied uniformly over the top, bottom and sides of the cable region, and also served as an encapsulating layer to help with insulation in the body. Diluted silicone adhesive for use with the PCB mould was prepared by adding $1.5 \mathrm{~mL}$ of xylene to $1 \mathrm{~g}$ of silicone adhesive in a sealed tube and subjecting it to sonication for $\sim 6 \mathrm{~h}$ until the silicone adhesive was completely dissolved. The PCB region of the GT-RE was placed in the PCB mould, immersed in dilute silicone adhesive, and subjected to vacuum for $\sim 1 \mathrm{~h}$ to remove any air bubbles. During the course of vacuuming, additional layers of diluted silicone adhesive were added to the PCB mould to account for the shrinking volume of the silicone adhesive as the xylene was removed. The diluted silicone adhesive would also shrink during the curing process, and this was an advantage, creating a more robust mechanical seal around protruding components of the GTRE from the PCB region. The dilute silicone adhesive was allowed to cure for a minimum of $24 \mathrm{~h}$ before the photoresist release layer on the PCB moulds was dissolved with ethanol. With slight mechanical force, the insulated and encapsulated PCB regions of the GT-REs were easily removed. Prior to encapsulating the PCB region, some of the GT-REs were coated with a 10$\mu \mathrm{m}$ layer of Parylene C. This step was avoided in later devices, as adhesion between the GT-REs was observed to be strongest with silicone adhesive. In order to protect the exposed microchannel electrodes from being coated in Parylene $\mathrm{C}$, the microchannel region was temporarily enclosed in a Parafilm envelope. Similarly, the gold pin connector region of the headcap was enclosed in Parafilm.

The silicone tubes with signal extraction wires were also filled with dilute silicone adhesive, which aided in mechanical protection of the signal extraction wires and provided an extra layer of insulation. Silicone adhesive (non-dilute) was also applied to the exterior of the silicone tubes in a ring shape $\sim 3 \mathrm{~cm}$ away from the PCB region, providing a tethering point during implantation, as discussed later.

Insulation of the signal extraction wires as they entered the Omnetics headcap followed a similar procedure as for the PCB region of the GT-RE. The headcap was placed in the headcap mould, immersed in silicone adhesive (non-dilute), and allowed to cure for a minimum of $24 \mathrm{~h}$. The photoresist release layer on the headcap mould was dissolved with ethanol, and, with slight mechanical force, the insulated and encapsulated headcap of the GT-RE was easily removed. It should be noted that the ends of the signal extraction wires connected to the headcap were already insulated with a proprietary process and epoxy (Omnetics Connector Corporation). It was not known how effective this process and epoxy would be, necessitating in-house insulation and encapsulation. As a final insulation measure, the GT-REs were coated with a final 5- $\mu$ m layer of Parylene C. The Parafilm enclosures around the microchannel region and gold pins of the headcap were removed, and the junction was protected with silicone adhesive. Note that the GT-REs were subjected to brief treatments with either air or $\mathrm{O}_{2}$ plasma immediately prior to all insulation and encapsulation steps for the PCB region and headcap, to aid with adhesion.

\section{In vitro functionality}

Previously recorded neural signals were used as an artificial signal source to evaluate GT-RE functionality. The GT-REs were placed 
with the electrode/microchannel region submerged in phosphate-buffered saline (PBS), with particular attention being paid to ensuring that the solution fully filled the microchannels. By use of a stripped audio cable dipped into the PBS, the previously recorded neural signals were played into the PBS, and monitored through the GT-RE electrodes integrated with the PCB hub for signal extraction wires.

\section{In vitro cytocompatibility}

In vitro cultures of lumbar DRGs were used to assess the cytocompatibility of the GT-RE post-fabrication. The culture methodology has been previously reported (Srinivasan et al., 2015). Briefly, unrolled GT-REs lacking the PDMS cover layer were placed at the bottom of tissue culture wells. No protein coatings of any kind, such as laminin, were used to help with cell adhesion or overall cytocompatibility. DRGs were explanted from P0 rat pups, the nerve roots were dissected away, and the DRGs were placed on the unrolled GT-REs at the entrance to the microchannels. During the first several hours of incubation, a thin layer of Dulbecco's modified Eagle's medium/F12 with $10 \%$ fetal bovine serum and $50 \mathrm{ng} / \mathrm{mL}$ nerve growth factor (Roche) was applied to prevent the DRGs from floating and allow adhesion to take place. Following this, the culture wells were filled with the same medium, which was replaced every 2 days, including the nerve growth factor.

The DRGs were cultured for a total of 2 weeks, at which point they were fixed with $4 \%$ paraformaldehyde in PBS for $20 \mathrm{~min}$ and washed three times with $1 \times$ PBS. To allow visual assessment of neurite outgrowth, Schwann cell migration, and overall cytocompatibility, the cultures were reacted with antibodies to the $160-\mathrm{kDa}$ neurofilament protein (1 : 500, mouse IgG1 NF160; Sigma) and S-100 (1 : 250, rabbit IgG S100; Dako) overnight at $4{ }^{\circ} \mathrm{C}$. This was followed by application of the secondary antibodies goat anti-mouse IgG1 Alexa 594 (1:220; Invitrogen) and goat anti-rabbit IgG Alexa 488 (1: 220; Invitrogen). Finally, cell nuclei were marked by incubation in 4',6-diamidino-2-phenylindole diluted to a concentration of $10 \mathrm{~mm}$ in PBS for $15 \mathrm{~min}$ at room temperature. The fluorescently labelled cells and nuclei were visualized with a Zeiss upright microscope, and the images were captured with an Olympus digital camera.

\section{GT-RE implantation, electrophysiology, and signal analysis}

To prepare the GT-RE for implantation, a silicone tube was placed around the rolled microchannels, acting as a nerve cuff for suturing. Stainless steel foil (Multipurpose 304 Stainless Steel Foil; thickness, 0.002 inches; McMaster-Carr) was then wrapped around the nerve cuff and connected to a ground wire acting as an implantable electrical shield for the GT-RE. The stainless steel wrap served as a large ground and pseudo-Faraday cage to help shunt electromyographic signals and other electrical artefacts to ground. There was also another short $2-\mathrm{cm}$ wire emanating from the headcap that was wrapped around a stainless steel screw used to secure the headcap to the skull. This served as another ground in the case of wire breakage. For sterilization purposes, the GT-RE was immersed in ethanol for $24 \mathrm{~h}$. A vacuum line was used to ensure that ethanol was drawn into and through the microchannels, and to prevent bubbles from remaining. After $24 \mathrm{~h}$, the GT-RE was transferred to sterile PBS and incubated for another $24 \mathrm{~h}$ to remove all traces of ethanol prior to implantation. The transfer took place without breaking a liquid environment, ensuring that air was not introduced into the microchannels.

The GT-RE was implanted in the rat sciatic nerve transection animal model $(n=4)$. All animal procedures were approved by and carried out in accordance with the Institutional Animal Care and
Use Committee at Emory University. In isoflurane-anaesthetized rats, the sciatic nerve was exposed by an incision through the distal part of the overlying biceps femoris muscle, and then transected at the mid-thigh. The GT-RE was attached to the proximal and distal segments of the cut nerve with 9-0 nylon sutures. The musculature was then re-apposed with resorbable 6-0 sutures, leaving the two silicone tubes containing the 34 signal extraction wires emerging from between the muscle flaps. The silicone tubes and attached headcap were burrowed subcutaneously to a midline incision at the top of the skull. Importantly, the silicone tubing was tethered, with nonresorbable 5-0 sutures, to the underlying musculature along its path to the skull, in order to provide strain relief during normal rat movement. These tethering points were located near the upper thigh, and also above the vertebral column near the pelvis, where the tethering created a gradual S-shaped curve in the tubing. At the midline incision on the skull, the periosteum was retracted with a periosteal elevator. Forty per cent phosphoric etch gel (Henry Schein) was applied to clean the skull surface, and six 0.5 -mm-diameter stainless steel screws were drilled into the skull. Screws were placed bilaterally, anterior to bregma, posterior to bregma, and posterior to lambda, just lateral to the midline. Next, the burrowed head stage was situated on the skull and affixed with ultraviolet-curable dental cement (Henry Schein), which was applied to the screws and the head stage. The skin was then wound-clipped around the headcap, forming a percutaneous connector for the electrophysiology experiments.

Electrophysiological experiments were performed 3 weeks after the GT-RE implantation. All recordings obtained with the GT-RE were made while the implanted rats were ambulating on a treadmill, in order to record 'naturally occurring' single-unit APs. This is in contrast to electrically evoked multi-unit activity, which requires stimulation of the nerve, and is not nearly as challenging or translatable in terms of assessing neural interfacing capability. The general electrophysiological methodology has been reported previously (Hamilton et al., 2011; Sabatier et al., 2011). Briefly, the rats were placed on a level, single-lane Plexiglas-enclosed treadmill $(53 \times 10 \times 14 \mathrm{~cm}$; Columbus Instruments, Columbus, OH, USA) set to a modest speed of $10 \mathrm{~m} / \mathrm{min}$. The implanted GT-RE was connected to amplifiers through the percutaneous headcap connector. One hundred seconds of recordings were taken, amplified by $\times 1000$ with a bandpass of $300-3000 \mathrm{~Hz}$, and digitized for off-line analysis. Additional filtering was performed in software from 400 to $3200 \mathrm{~Hz}$ with a four-pole Butterworth filter. All recorded signals were sampled at $10 \mathrm{kHz}$. Single-unit APs were identified and sorted with the publicly available semi-automated spike-sorting algorithm Wave_ clus (Quiroga et al., 2004).

The number of microelectrodes that recorded single-unit APs was used to determine the overall electrode yield of the GT-RE. To ensure that the identified single-unit APs were not, in fact, the same AP being recorded by multiple electrodes, a cross-talk screen was performed. The logic for this cross-talk screen stems from the fact that, if the same AP is recorded on multiple electrodes, the AP will occur at the same time across those electrodes. In this case the occurrence or timestamps of this AP on any one electrode will match its timestamps on the other electrodes. Mathematically speaking, the timestamps for the AP from the different electrodes would match each other and, at a minimum, overlap by at least $50 \%$.

On the basis of this logic, 1-ms time windows centred on the timestamps of an AP recorded with a given electrode in a device were compared with the timestamps of all other APs identified across all other electrodes of the same device. One-millisecond time windows were used instead of the actual timestamp to help account 
for any small differences in timestamps that might occur for a variety of reasons. Any APs identified across multiple electrodes that occurred together by $>50 \%$ on the basis of their timestamps were then visually inspected to determine whether the waveforms were also similar, indicating that they may be the same AP. The AP waveforms were visually analysed for similarity in shape, excluding differences in amplitude and numbers of occurrences. Similarity in amplitude was not used as determinant criterion of cross-talk, because if an AP acquired from a given electrode also appeared on another through cross-talk, it would probably appear to be reduced. The number of occurrences of the single-unit APs was also not used as a determinant criterion, on the basis of similar logic. The number of occurrences of an AP could, at most, be the same, if not probably lower, on the receptive electrode than on the source electrode conducting the true signal. If it was determined that, across two or more electrodes, the waveform shapes were visually identical, the AP was then assigned to the electrode that recorded the largest average amplitude and the greatest number of occurrences of that waveform.

\section{Results}

\section{GT-RE fabrication}

An image of the Parylene C MEA with patterned SU-8 microchannel walls is shown in Fig. 4A. The de-insulated gold electrodes and insulated gold traces can also be seen. The gold traces were $30 \mu \mathrm{m}$ in width, and the gold electrodes were $80 \times 40 \mu \mathrm{m}$. The SU-8 microchannels were $20 \mu \mathrm{m}$ in width and $100 \mu \mathrm{m}$ in height, which cannot be seen in this image, and $3.5 \mathrm{~mm}$ in length. A side view of the microchannels after the PDMS capping layer had been applied is shown in Fig. 4B. The microchannels are clearly visible, along with the $100-\mu \mathrm{m}$-tall SU-8 microchannel walls. The Parylene C substrate and PDMS capping layers are also visible. Figure $4 \mathrm{C}$ is an image of the microchannels after rolling resulting in a microchannel conduit of $\sim 1.5 \mathrm{~mm}$ in diameter. The Parylene $\mathrm{C}$ cable extending from the microchannel to the bonding pads (outside the image) can be seen to the right of the microchannel conduit. The GT-RE after addition of the signal extraction wires and encapsulation is shown in Fig. 4D. In these images, the stainless steel foil wrap has been omitted to allow visualization of the microchannel region. A top-down view and side view are both shown.

\section{In vitro functionality}

The GT-RE microelectrodes were functionally tested in vitro for their ability to be used to appropriately detect neural signals. The microelectrodes had a mean impedance of $640 \mathrm{k} \Omega$ measured at $1 \mathrm{kHz}$. Evaluation of the ability to record neural signals was accomplished by recording an audio neural signal played into PBS containing the GT-RE. A schematic of the setup is shown in Fig. 5A. An example of the recorded audio neural signals, along with a magnified portion showing single-unit APs, taken with the GT-RE is shown in Fig. 5B. This recording was obtained from a single microelectrode of the GT-RE. Single-unit APs as small as $\sim 30 \mu \mathrm{V}$ in amplitude were successfully recorded. Microelectrodes with traces that were physically cut on purpose did not result in successful recordings of the audio neural signals, as expected.

\section{In vitro cytocompatibility}

DRGs were cultured on unrolled GT-REs lacking the capping PDMS layer to assess overall cytocompatibility for a PNI applica-
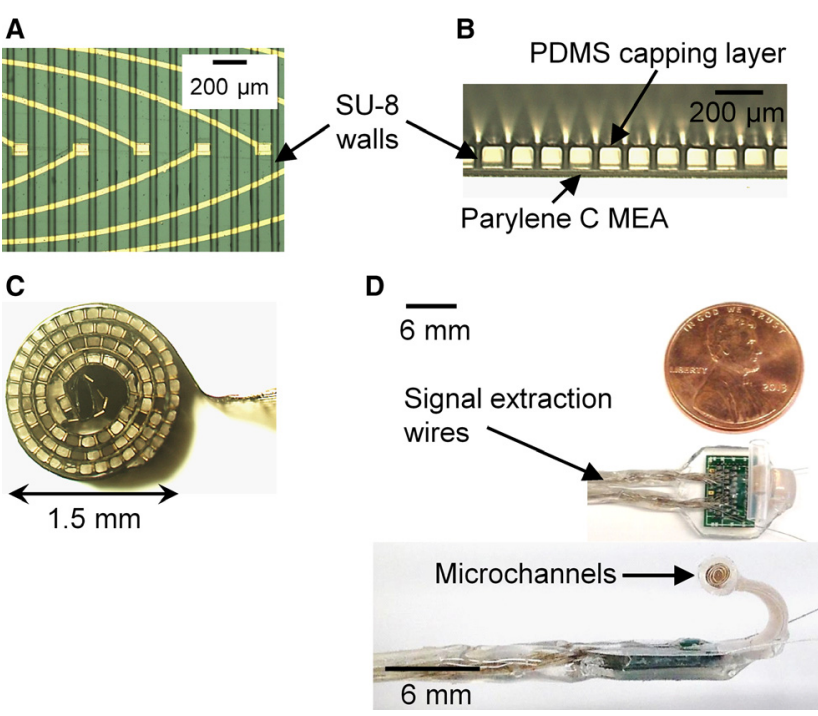

FIG. 4. GT-RE fabrication. (A) Image with a top view of the Parylene C MEA after metal patterning, Parylene $\mathrm{C}$ passivation layer patterning, and addition of the SU-8 microchannel walls. Five microelectrodes are viewable in this image. (B) Image with an entrance view of the microchannels after the PDMS capping layer has been applied prior to rolling. The SU-8 microchannel walls and Parylene $\mathrm{C}$ substrate are viewable as well. (C) Image with an entrance view of the microchannels after rolling. The resulting microchannel cylinder is $\sim 1.5 \mathrm{~mm}$ in diameter. (D) Images of the GT-RE after hermetic encapsulation and packaging. Images include a top-down view showing the surface mount PCB with signal extraction wires, and a side view in which the microchannel region is visible. Note that the microchannel region is curved over the PCB to reduce the overall footprint of the device for implantation.
A

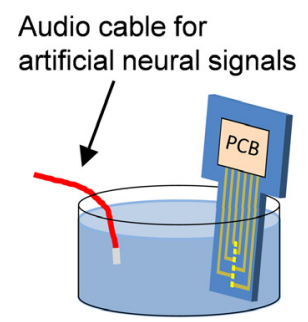

8

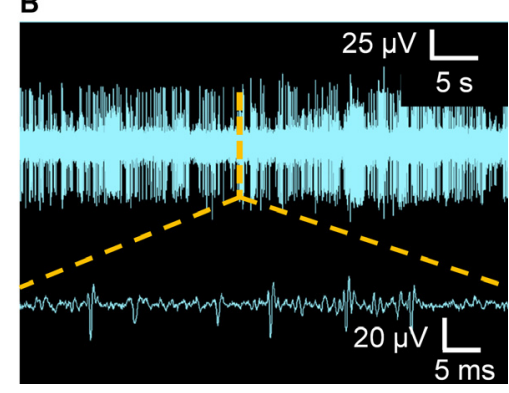

FIG. 5. In vitro functional testing with artificial neural signals. (A) Schematic of the in vitro setup, in which an audio cable was used to play artificial neural signals into PBS containing the GT-RE. (B) Example recording of the artificial neural signal taken with the fully integrated GT-RE, validating functionality. Single-unit APs of the order of $30 \mu \mathrm{V}$ can be observed in the bottom magnified trace.

tion. In general, the DRGs adhered well where they were initially placed at the entrance to the SU-8 microchannels. A representative DRG culture stained for axons (red) and Schwann cells (green) is shown in Fig. 6A. The electrodes and traces are the curved darker vertical lines, and the SU-8 microchannel walls are the faintly fluorescent horizontal lines. The DRG is located on the left side of the microchannels. Portions of the image appear overexposed, owing to the high degree of cellular growth. Despite the intense fabrication process and exposure to toxic chemicals, the GT-REs were readily capable of supporting multicellular growth emanating from the DRGs. Both axons and Schwann cells grew and proliferated in a highly robust manner past the end of the microchannels in 


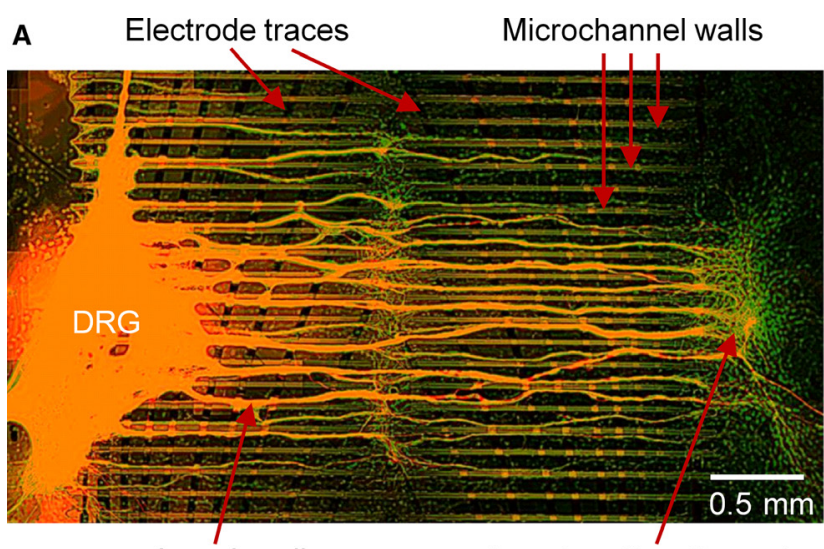

Axon bundle

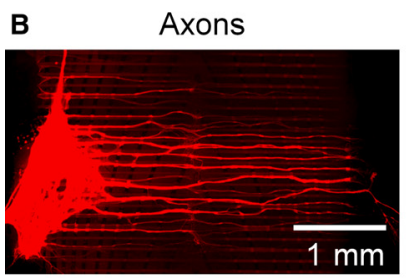

Axon bundles dispersing

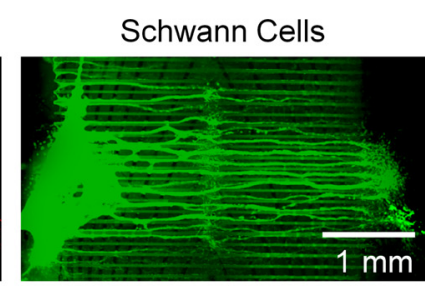

C Electrode traces

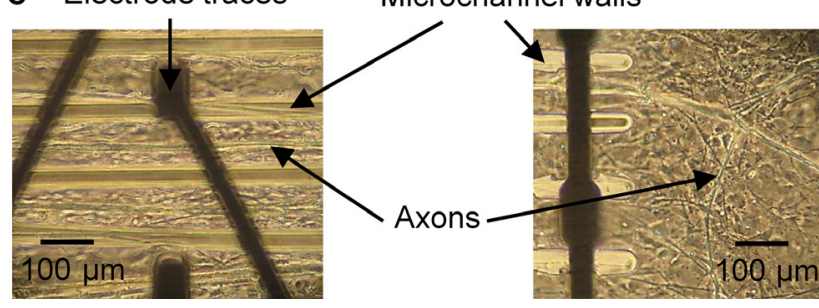

FIG. 6. In vitro cytocompatibility studies on the GT-RE. (A) DRG in vitro culture with axon processes and migrating Schwann cells on 'open' $100-\mu \mathrm{m}-$ wide microchannels of the GT-RE. Axon bundles and Schwann cells observed emanating from the DRG were aligned and oriented in the microchannels. The axons and Schwann cells grew/migrated out past the 3.5mm-long microchannels, where the axons bundles appeared to disperse. Red: axons. Green: Schwann cells. Orange: DRG and regions of axon/Schwann cell co-localization. Microchannels are visible as dark horizontal regions separated by faintly autofluorescent SU-8 walls. Electrode traces are visible as vertically curving lines. (B) Unmerged fluorescent images of axons and Schwann cells from A. (C) Bright-field images of axons and Schwann cells at the electrode region of the microchannels and at the end of the microchannels. A sheet of Schwann cells is present over the entire substrate, making individual cell identification difficult.

$<2$ weeks. In general, axon extension and Schwann cell migration were aligned and oriented in the direction of the microchannels, and also appeared to be preferential towards the microchannels. The degree of extension and migration towards the microchannels was so extreme that the bulk mass of the DRGs actually moved from the original location in front of the microchannels onto the microchannels.

Axons emanating from the DRGs formed bundles of axons similar in nature to fascicles found in nerves in vivo. For the most part, these bundles stayed within a single microchannel, but at times could be observed growing over the microchannel wall into a neighbouring microchannel. However, the growth continued in a general unidirectional manner. Regardless of the microchannel, the axons and Schwann cells seemed to be as equally likely to grow on the Parylene C substrate as on the SU-8 microchannel walls. It was also observed that the axon bundles tended to disperse at the end of the microchannels (Fig. 6A). It was difficult to fluorescently image the Schwann cells, owing to the large degree of proliferation and resulting fluorescence intensity. The exposure time had to be significantly reduced as a result, making the degree to which Schwann cells are present seem significantly less than in reality. Bright-field images of the microchannel region and exit side of the microchannels are included in Fig. 6B to illustrate the striking degree of proliferation. A 'sheet' of Schwann cells was present on the surface of the GT-RE.

\section{In vivo electrophysiology}

All GT-REs, with an average of 19 electrodes $(63.33 \%) \pm 3.9$ standard deviations, successfully recorded a multitude of unique singleunit APs 3 weeks after implantation in an awake and ambulating scenario. As noted in Materials and methods, single-unit APs were deemed to be unique on the basis, first, of a comparison of the timestamps of the APs. If the occurrence of different APs overlapped by $>50 \%$, the waveforms were visually assessed for similarity in shape. If they were deemed to be similar, the AP was assigned to a single electrode to prevent double counting of the AP across two or more microelectrodes. The average peak-to-peak amplitude of the APs was $\sim 125 \mu \mathrm{V}$, with a $77-\mu \mathrm{V}$ standard deviation. The average signal-to-noise ratio (SNR) was calculated by dividing the peak-to-peak amplitude of each AP by the peak-to-peak noise band (two times the standard deviation) and averaging the resulting individual SNRs. This was found to be 2.98 , with a standard deviation of 1.4. Note that the APs were not subtracted out or removed from the raw signal for noise band calculation. In some cases, the presence of large artefacts during a given trial affected the noise band calculation. These trials were not included in the analysis. Figure 7 shows a stereotypical raw signal recorded with an electrode from a single GT-RE. Identified single-unit APs from the raw signal along with the respective spike raster plots are also shown. In many cases, multiple single-unit APs could be identified in a recording from a single electrode. However, spike sorting to identify all possible single-unit APs recorded with every electrode was not performed, owing to the time-intensive nature of the analysis. Figure 8 shows an example GT-RE and all of the microelectrodes integrated in the microchannels that were successfully used to record single unit APs. Examples of the APs from all of the microelectrodes are shown alongside the MEA image. As mentioned previously, all recordings were performed while rats were ambulating on the treadmill.

An evaluation of the success of each microelectrode in recording an AP based on spatial location within the cross-section of the GTRE was also performed. Each microelectrode position was taken, and for the four GT-REs, evaluated for the percentage success in recording an AP. This is depicted as a colour map in Fig. 9A, where solid green, light green, light red and solid red indicate the given microelectrodes that recorded an AP in 100, 75, 50 and $25 \%$ of the GT-REs, respectively. A statistical analysis with a two-tailed paired $t$-test was performed, comparing the average percentage success of recording an $\mathrm{AP}$ with the innermost 15 microelectrodes vs. the outermost 15 microelectrodes. This is shown in Fig. 9B, where the inner half of the microelectrodes in the GT-RE were, on average, $15 \%$ more successful $(P=0.027)$ at recording APs than the outer half of the microelectrodes.

Although all GT-REs were successful in recording a multitude of single-unit APs at week 3, by week 6 the Parylene C passivation showed signs of delamination from the Parylene $\mathrm{C}$ substrate layer in the cable region of the GT-RE. This is shown in Fig. 10, where 


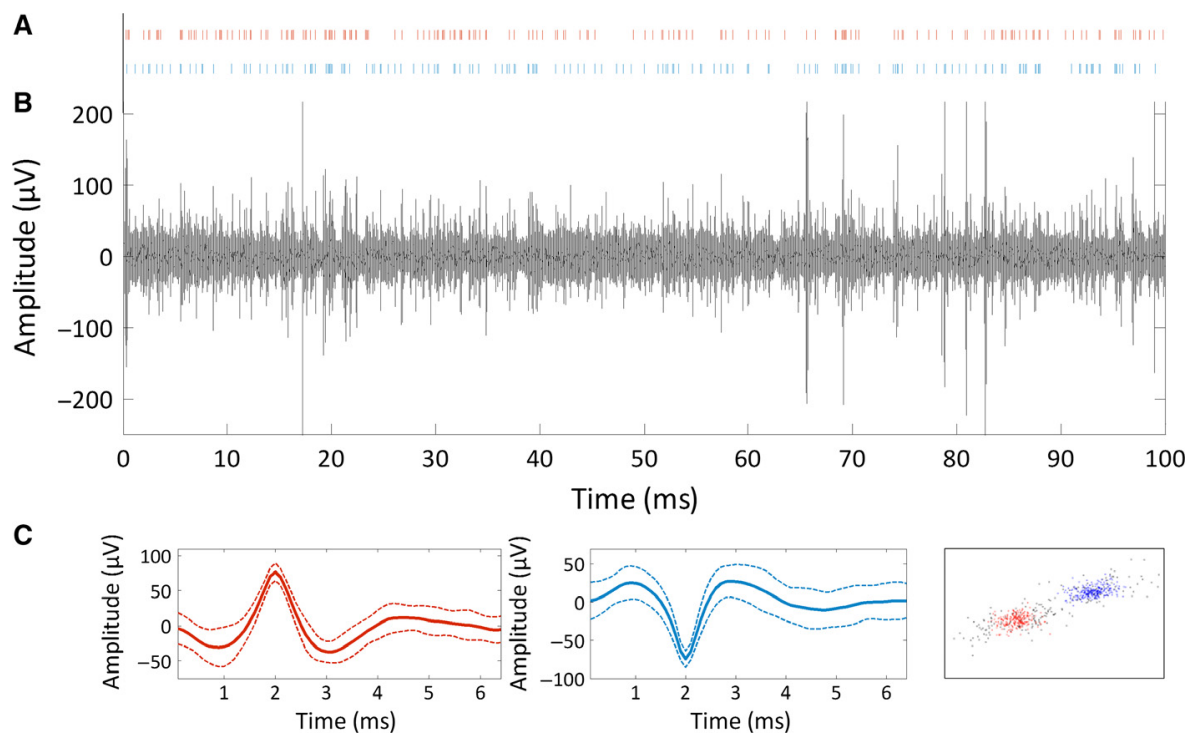

FIG. 7. GT-RE in vivo stereotypical recording in ambulating rats. (A) Spike raster plots of two identified APs from the raw signal shown in B. (B) Example raw recording signal obtained over a period of $100 \mathrm{~s}$ from a single microelectrode of a GT-RE. (C) Waveforms and clusters of two single-unit APs identified in the raw signal. Dotted lines correspond to $\pm 1 \mathrm{SD}$ from the mean. Colours correspond to the raster plots in A.
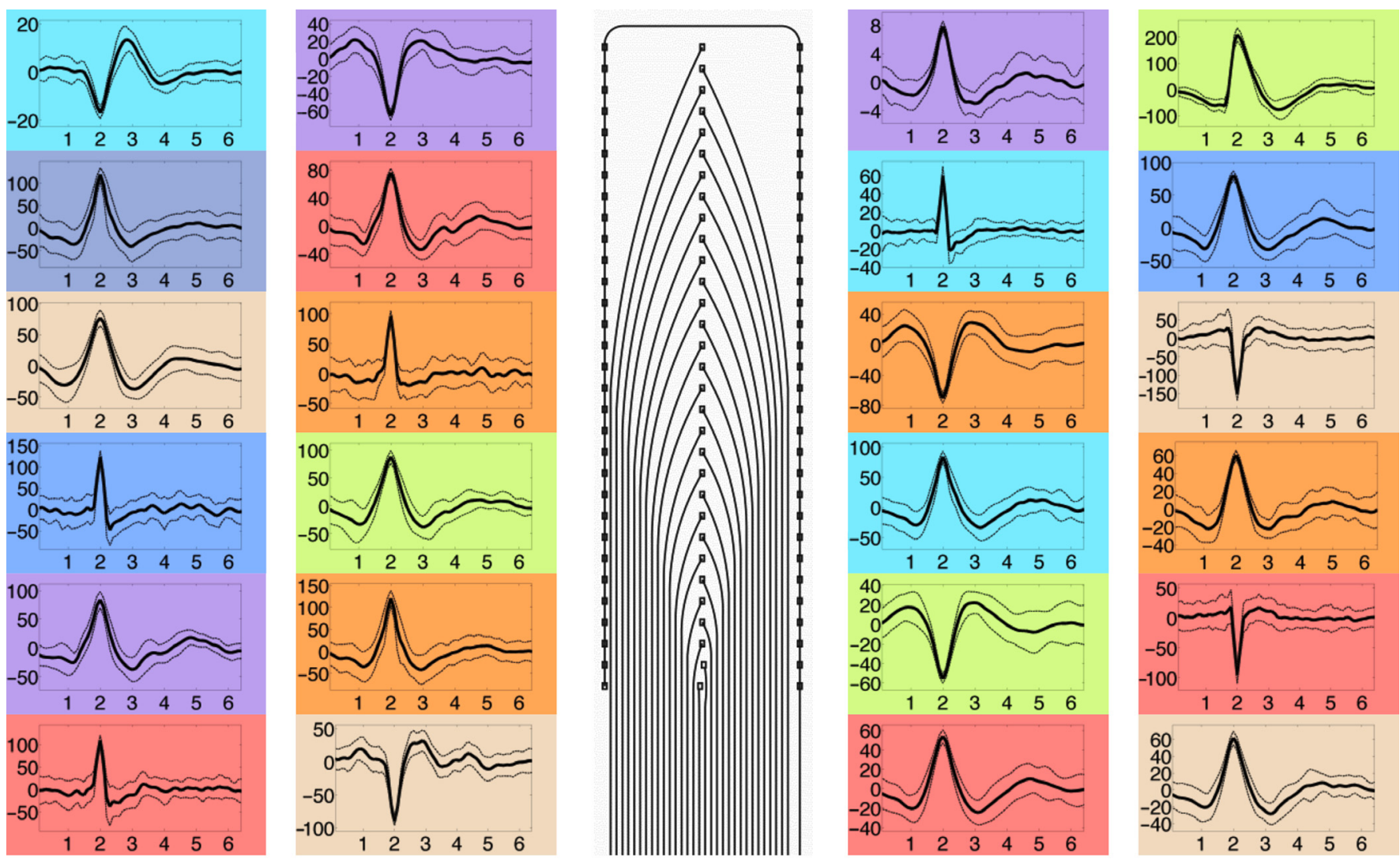

Amplitude $(\mu \mathrm{V})$

Time (ms)

FIG. 8. Collage of AP waveforms recorded with a GT-RE in an ambulating rat. Example waveforms were recorded with a single GT-RE. All APs were recorded with different electrodes from the GT-RE. In this case, a total of 24 APs were identified with 24 different electrodes within the GT-RE. Dotted lines correspond to $\pm 1 \mathrm{SD}$ from the mean. The microelectrode schematic is shown in the middle for reference. 

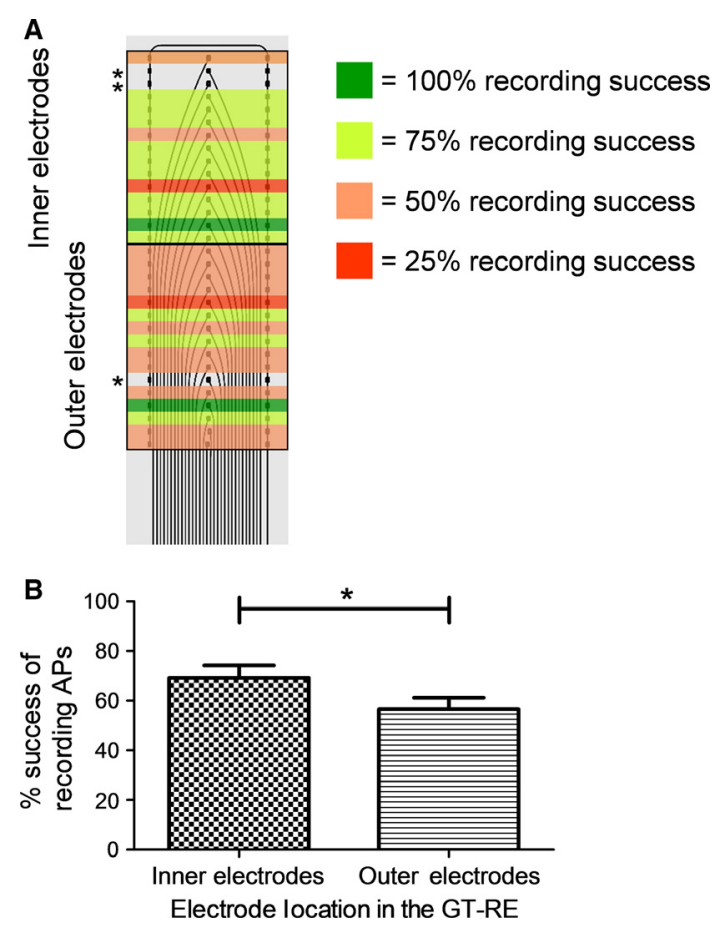

FIG. 9. Recording success of inner microelectrodes as compared with outer microelectrodes across all GT-REs in ambulating rats. (A) Colour map of the percentage success of recording an AP for each microelectrode across all GT-REs. Solid green indicates the given microelectrode that successfully recorded an AP in all GT-REs. Light green indicates the given microelectrode that recorded an AP in 75\% of the GT-REs. Following from this, light red indicates $50 \%$ of the GT-REs and solid red indicates 25\% of the GTREs. *Microelectrodes that were not connected during recordings and were therefore not included in the analysis. (B) Quantitative analysis of the average percentage success of recording an AP with the inner electrodes of the GT-RE as compared with the outer electrodes of the GT-RE. On average, the microelectrodes in the inner half of the GT-RE were statistically more successful at recording an AP than those in the outer half. Mean \pm standard error of the mean, two-tailed paired $t$-test, ${ }^{*} P<0.05$.

forceps were inserted between two previously adhered Parylene C layers after they were physically separated. Therefore, recordings were not analysed at the 6-week time point. However, it is important to note that, whereas the Parylene $\mathrm{C}$ showed signs of delamination, the silicone adhesive used for encapsulation did not show any signs of delamination, despite extensive physical attempts to cause visible delamination of the silicone adhesive.

\section{Discussion}

The vast majority of commercially available advanced prosthetic limbs provide only a fraction of the functionality of the natural limb that they are meant to replace. This shortcoming largely stems from an individual's inability to 'control' the advanced prosthetic. As a result, there remains a need to design interfaces that enable seamless integration between conscious thought and resulting prosthetic limb movement for amputees. Peripheral nerve interfacing has the potential to provide such integration by obtaining neural signals from an amputated nerve that are intended for muscle activation and natural limb movement. These biological signals can then be translated into digital input for robotic limb control. Furthermore, there is the opportunity to implant a PNI at the time of surgery in cases of amputation or nerve repair.

In the last 5 years, there have been significant advances in establishing the groundwork for microchannels as a viable avenue to
A

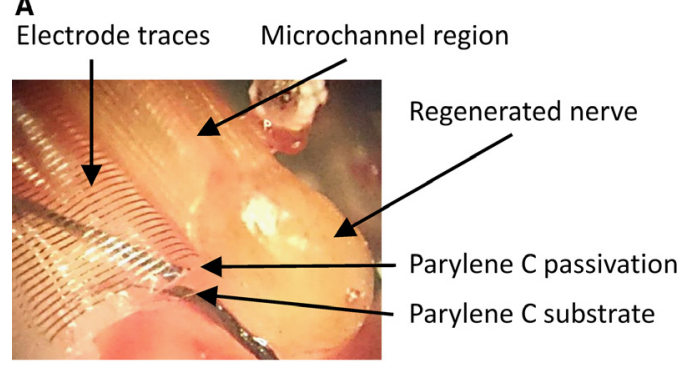

B

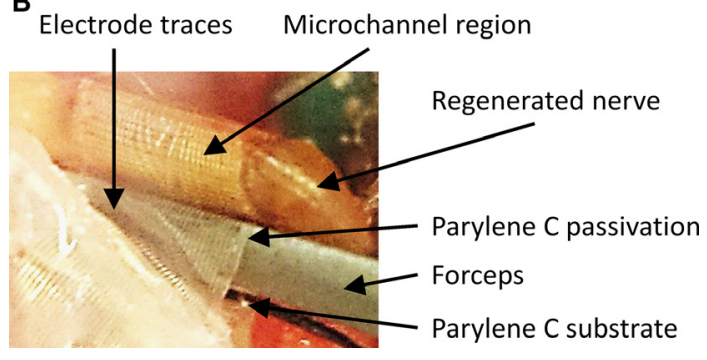

FIG. 10. Parylene C delamination. (A) Example of delamination in the Parylene $\mathrm{C}$ cable between the passivation and substrate layers after 6 weeks of implantation. The rolled microchannel region with the regenerated nerve is clearly visible. Gold traces in the Parylene $\mathrm{C}$ cable leading from the microchannel region to bonding pads (out of image) are also clearly visible. (B) Forceps have been inserted between the Parylene C passivation layer and Parylene $\mathrm{C}$ substrate layer to aid in identifying the region of delamination.

chronic peripheral nerve interfacing. Acute non-regenerative implementations of microchannels have been evaluated, whereby dissected nerve strands were placed in microchannels and recordings were performed (Delivopoulos et al., 2012; Minev et al., 2012; Chew et al., 2013). These studies validated the potential for microchannels to be used in vivo for single-unit AP recordings. Other studies implanted microchannel interfaces and performed evaluations after regeneration of transected nerves through the microchannels (FitzGerald et al., 2012; Gore et al., 2015; Srinivasan et al., 2015). These studies validated the potential for microchannels to be implemented chronically, and the potential to record single-unit APs after chronic implantation. To date, however, fully integrated, large electrode count microchannel interfaces have not been implemented and evaluated in awake and freely moving rodents. Although this is arguably the most challenging experimental model for evaluating a PNI, it is a necessary step towards clinical translation.

Here, we have designed, fabricated and evaluated a 30-microelectrode microchannel interface, the GT-RE, for its ability to record single-unit APs in awake and ambulating rodents. First, the functionality of the device was validated in vitro by functional testing and DRG cytocompatibility studies. Finally, the GT-RE was evaluated in vivo to assess the potential for chronic peripheral nerve interfacing in the rat sciatic nerve animal model. The GT-RE was designed as a sheet of 97 parallel microchannels with gold microelectrodes integrated within the base at the centre of the microchannels. The sheet of microchannels was rolled to form a spiral conduit that matched the shape and size of the rat sciatic nerve. This design allowed standard microfabrication and lithography techniques to be used to fabricate the device. The microelectrodes themselves were connected to bonding pads via small gold traces in a cable that was then connected to a surface mount PCB acting as a hub for signal extraction wires.

In vitro functional testing was utilized to assess the capability of the GT-RE to record single-unit APs similar in size and nature to those that would be encountered in vivo. The GT-RE was successfully used to record artificial neural signals injected into PBS on the 
order of $30 \mu \mathrm{V}$. An example recording from a single electrode of the GT-RE is shown in Fig. 5B. The ability to record the artificial neural signals through the GT-RE showed that the microelectrodes integrated into the GT-REs were functional. Additionally, the GT$\mathrm{RE}$ could be used for neural recordings of some of the smallest APs commonly observed in vivo.

Cytocompatibility studies revealed that there is no apparent toxicity arising from the fabrication processes used to produce the GT$\mathrm{RE}$, and that the GT-RE is suitable for implantation. Data were obtained without any surface coatings or treatments of any kind, including plasma treating to increase hydrophilicity or protein coatings such as laminin to facilitate adhesion and cytocompatibility. In fact, the axon growth and Schwann cell proliferation were so robust that imaging became extremely challenging, owing to the overall levels of fluorescence and the resulting appearance of overexposure (Fig. 6A). The Schwann cells, in particular, proliferated to a degree that was largely unexpected, as they formed a sheet of cells across the substrate (Fig. 6B). Interestingly, the axons formed bundles similar to fascicles observed in vivo and reminiscent of those observed in a previous regeneration study in which microchannel fascicles were described (Fig. 6A) (Srinivasan et al., 2015). These axon bundles grew out past the 3.5-mm-long microchannels, and would probably have continued if the microchannels were longer. This was previously observed in terms of axon length, where growth occurred out to $4 \mathrm{~mm}$ in just 7 days, even though the axon bundles were not present (Srinivasan et al., 2015). It does appear, however, that, without the presence of the microchannel topography, the axon bundles did not continue to grow further, but instead disassembled, and the axons congregated near the exit of the microchannels (Fig. 6A). This suggests that the formation of the axon bundles is inherently tied to the presence of the microchannels. Furthermore, the formation of axon bundles in this study as compared with the lack thereof in the previous studies is attributed to the $100-\mu \mathrm{m}$-wide microchannels used here, as opposed to the $50-\mu \mathrm{m}$-wide or $75-\mu \mathrm{m}$-wide microchannels used previously (Srinivasan et al., 2011, 2015). The 100- $\mu$ m-wide microchannels used here also match those used in vivo in the previous study, in which uniform fascicular growth characterizing 'microchannel fascicles' was observed (Srinivasan et al., 2015). This further supports the hypothesis that, in vitro, axon bundle formation is a microchannel structure-based phenomenon. In contrast, axon bundle formation is unlikely to be a material-based phenomenon of Parylene C vs. PDMS, as used previously, because both have been established to be cytocompatible in general, and with DRGs in particular. Adhesion of DRGs and associated proliferating/migrating cell types was also observed on both substrates, further indicating a lack of differences stemming from material selection.

It was also interesting to observe that the mass of the DRGs cultured on the GT-REs in all cases actually migrated from their original position closer to or even onto the microchannels. Specific attraction to the SU-8 microchannel walls does not appear to explain this, however, as it was noted earlier that the axon bundles did not seem particularly inclined to grow on the SU8 as compared with the Parylene C. Despite the lack of material preference for growth or proliferation, the axons and Schwann cells were aligned and oriented to the direction of the microchannels, as expected. It is also worth noting that any axon growth and Schwann cell proliferation on top of the microchannel walls is not relevant to the in vivo application, as there would be a capping PDMS layer forcing the axons and Schwann cells to remain within the microchannels.

The GT-RE implants were tolerated well by the rats, with no additional discomfort other than that stemming from the initial surgery. However, the GT-RE did, in many ways, fill the cavity below the biceps femoris muscle, and significant increases in PCB size would be difficult to accommodate, at least in a rat animal model. Placing the rolled microchannel region over the middle of the PCB was a significant advantage, as it allowed the PCB to be situated deeper into the leg of the rat, and is recommended for future experiments. A 3-week time point was chosen after implantation for electrophysiology recordings to allow enough time for axons from the proximal end to regenerate into and probably through the microchannels. Data from an unpublished 4-week study showed ample regeneration in similarly sized microchannels.

Successful electrophysiological recordings were obtained from the GT-RE at a 3-week time point. An example of a raw signal from a stereotypical microelectrode within a microchannel is shown in Fig. 7B. A multitude of single-unit APs were identified within this and other raw signals across the many microelectrodes from the GTRE. Examples are shown in both Figs 7 and 8. Overall, 63\% of the microelectrodes integrated into the microchannels of the GT-RE were successfully used to record single-unit APs. This is a significant finding, considering previously reported success rates with other microchannel interfaces, and especially given that the GT-RE is a large electrode count microchannel interface with 30 integrated microelectrodes (Delivopoulos et al., 2012; FitzGerald et al., 2012; Minev et al., 2012; Chew et al., 2013; Srinivasan et al., 2015). The single-unit AP waveforms, amplitudes and SNRs reported here also fell within ranges previously reported in studies using microchannels in acute scenarios or terminally after implantation (Delivopoulos et al., 2012; FitzGerald et al., 2012; Minev et al., 2012; Chew et al., 2013; Srinivasan et al., 2015). Furthermore, the GT-RE enabled successful recordings in awake and ambulating animals, building significantly on these previous acute and terminal experiments. The ability to record and identify single-unit APs is the paramount criterion by which PNIs are judged for a host of clinical applications, from bladder control to robotic limb control. Accordingly, the findings reported here give significant credence to further work on developing these technologies, which have the eventual target of enabling clinical application.

Interestingly, it was found that the inner microelectrodes of the GT-RE were, on average, more successful at recording single-unit APs than the outer microelectrodes, as shown in Fig. 9A and B. A previous study evaluating the regeneration of axons through microchannel scaffolds noted that microchannels lacking axon regeneration tended to be located near the periphery of the device (Srinivasan et al., 2015). Given the early 3-week time point used in this study, as compared with the 8-week time point in the previous study, this effect could have been exacerbated here, as regeneration would not yet have stabilized, and could still be occurring in the outer microchannels. Myelination would also be ongoing, and may be lagging in the outer microchannels. The net effect would be reduced AP recording success with the outer microelectrodes. That being noted, it is expected that the difference in AP recording success between the inner and outer microelectrodes will diminish over the following weeks and months as the regeneration stabilizes. These findings are also important from a design perspective, as researchers performing microchannel interfacing at earlier time points, such as 3 weeks, should bias microelectrode placement towards the centre of the device.

Given the 3-week time point used for these electrophysiology recordings and the fact that the axons had to traverse a 3.5-mm gap before reaching the distal segment, it is highly unlikely that these axons reinnervated their distal targets. Therefore, this early recording time point is, in some ways, similar to a nerve amputee animal model, in which the amputated nerve permanently lacks its distal 
reinnervation targets. Therefore, the success in recording a multitude of single-unit APs at this early time point can be used as an indicator that microchannel interfacing in an amputee scenario would also probably be successful. This is especially true given previous studies that have investigated and shown the regenerative capacity and stability of amputated nerves once they have grown into and through microchannel devices (Srinivasan et al., 2015). It can also be surmised that a majority of the single-unit APs recorded were probably motor units, because the input for most sensory units would be in the distal targets that had yet to be reinnervated. Therefore, the total number of APs recorded was probably significantly lower than what could be recorded at future time points once reinnervation occurs and sensory input generates additional APs. This may, in part, account for the electrodes that did not successfully record single-unit APs. Of course, recording sensory APs is only relevant to scenarios in which sensory input is of value. For advanced prosthetic control in particular, the number of reliably recordable motor APs is the first primary concern. However, recording sensory APs and utilizing conduction-blocking techniques holds significant value for mitigating the chronic and often debilitating pain that individuals experience post-amputation, in addition to providing sensory feedback through prosthetics.

Whereas there was significant success at the 3-week time point with the GT-RE, delamination issues in the cable region of the GTRE were observed within the device at a 6-week time point. An example of the delamination in the cable region is shown in Fig. 10. The rolled microchannel region with the regenerated sciatic nerve and the microelectrode traces leading to the bonding pads (outside the image area) can also be seen in Fig. 10. The fact that delamination occurred in the GT-RE was unexpected, given previous reports indicating the utility of Parylene $\mathrm{C}$ as a viable chronic substrate and insulator for in vivo applications with flexible cortical and retinal interfacing devices (Pang et al., 2005; Rodger et al., 2006, 2008; Hassler et al., 2011; Kim et al., 2013). It has even been shown by multiple studies using accelerated failure testing that Parylene C should perform as a viable insulator for up to or longer than 20 years of implantation (Li et al., 2006, 2008, 2010; Hsu et al., 2009). It seems evident that, at least in the context of peripheral nerve interfacing, these accelerated failure tests must be viewed with significant caution. One possible reason for the large discrepancy is that the accelerated failure tests are most often performed in saline baths. This does not accurately represent the blood-filled environment, where numerous immune cells and components of the blood play active roles in attempting to degrade an implant. These processes are integral parts of device failure, in this case delamination. Another potential cause for the discrepancy is the lack of mechanical or physical stress/movement during the accelerated failure tests. In these tests, the devices are stationary and not subject to the motions typical of an in vivo environment, especially that of the limbs. This is also partly true of cortical and retinal interfacing, which occur in less movement-prone regions and therefore subject the devices to less physical or mechanical stress. In fact, inspection of the GT-RE showed numerous areas of buckling and plastic deformation of the Parylene $\mathrm{C}$ in areas of high movement, along with delamination occurring in those regions, indicating that physically/ mechanically induced failure played a role in overall device failure.

Whereas Parylene C-Parylene C delamination was observed at the 6-week time point, adhesion of the silicone adhesive used to encapsulate the GT-RE was intact. Attempts to physically remove the silicone adhesive were unsuccessful, indicating strong bonding and resistance to delamination. It is also worth mentioning that Parylene $\mathrm{C}$ is fundamentally only flexible as a two-dimensional film.
Application over a three-dimensional object that can flex, e.g. a tube, causes buckling of the Parylene $\mathrm{C}$ at the region of bending and/or plastic deformation on the opposite side, owing to its inability to stretch. Both of these result in stress points within the Parylene $C$ film, and will eventually cause failure of the Parylene $C$ after repeated motion. Silicone derivatives do not suffer from this disadvantage, and may therefore hold significantly more promise with devices that are subject to mechanical stress and/or trauma during their lifetime. It is therefore recommended that future studies should investigate the use of dilute forms of the silicone adhesive as a substrate for device fabrication, in addition to being used for encapsulation purposes. The fabrication of such devices should not be significantly different from fabrication on PDMS. In the end, the fact that the GT-RE eventually suffered from delamination issues indicates a need for further materials development with regard to hostile in vivo environments. Once these adhesion issues are mitigated in the in vivo environment, future studies can investigate truly chronic AP recording capabilities by using the GT-RE or other microchannel interfaces. This, however, does not diminish the significant promise shown by recording single-unit APs from the large number of integrated microelectrodes at the 3 -week time point. The ability to use a fully integrated microchannel interface, the GT-RE, for regenerative peripheral nerve interfacing in awake and ambulating rodents has been established. Future studies will continue to build upon these findings, bringing microchannel interfacing closer to clinical translation.

\section{Conclusion}

In this study, a novel microchannel interface, the GT-RE, with large numbers of integrated microelectrodes was evaluated in a challenging, awake and freely moving rat animal model. In vitro functional testing validated the capability of the GT-RE to record single-unit APs similar in size to those found in vivo. DRG in vitro cultures revealed striking cytocompatibility of the GT-RE post-cleanroom processing with large degrees of axon growth and Schwann cell proliferation. The characteristics of the growth even resembled those of the nerve fascicles normally found in vivo. Finally, in vivo implantation of the GT-RE revealed the capability of microchannel interfacing to be utilized for high-throughput single-unit AP recording in awake and ambulating rats. On average, $63 \%$ of the 30 microelectrodes per device were successfully utilized for this as early as 3 weeks post-implantation, marking a significant advance in microchannel interfacing. At 6 weeks, delamination of the substrate materials for the GT-RE was observed, indicating a need for future materials development to enable chronic recordings. In vivo observations indicate that silicone derivatives as substrate materials, in addition to an encapsulant, may be the way forward. In the end, the findings of this study will help to direct future technology development and enable truly chronic, preclinical testing of peripheral nerve interfacing with the relatively new microchannel approach.

\section{Acknowledgements}

The authors would like to acknowledge support from the Defense Advanced Research Projects Agency (DARPA) MTO under the auspices of Dr Jack Judy through the Space and Naval Warfare Systems Center Pacific Grant/ Contract No. N66001-11-C-4167, and the European Research Council through a Starting Grant/ERC 259419 ESKIN to S. P. Lacour. The authors would also like to thank Bill Goolsby for PCB design support, Dr Jill Ward and Benjamin Huey for animal surgery support, Dr Nassir Mokarram for manuscript support and discussions, and Dr Balakrishna S. Pai for support with the scientific principles employed in this study. 


\section{Abbreviations}

AP, action potential; DRG, dorsal root ganglia; GT-RE, Georgia Tech. Regenerative Electrode; MEA, microelectrode array; PBS, phosphate-buffered saline; PCB, printed circuit board; PDMS, polydimethylsiloxane; PNI, peripheral nerve interface; SNR, signal-to-noise ratio.

\section{References}

Branner, A. \& Normann, R.A. (2000) A multielectrode array for intrafascicular recording and stimulation in sciatic nerve of cats. Brain Res. Bull., 51, 293-306.

Branner, A., Stein, R.B. \& Normann, R.A. (2001) Selective stimulation of cat sciatic nerve using an array of varying-length microelectrodes. J. Neurophysiol., 85, 1585-1594.

Branner, A., Stein, R.B., Fernandez, E., Aoyagi, Y. \& Normann, R.A. (2004) Long-term stimulation and recording with a penetrating microelectrode array in cat sciatic nerve. IEEE Trans. Biomed. Eng., 51, 146-157.

Chew, D.J., Zhu, L., Delivopoulos, E., Minev, I.R., Musick, K.M., Mosse, C.A, Craggs, M., Donaldson, N., Lacour, S.P., McMahon, S.B. \& Fawcett, J.W. (2013) A microchannel neuroprosthesis for bladder control after spinal cord injury in rat. Sci. Transl. Med., 5, 210ra155.

Clements, I.P., Mukhatyar, V.J., Srinivasan, A., Bentley, J.T., Andreasen, D.S. \& Bellamkonda, R.V. (2013) Regenerative scaffold electrodes for peripheral nerve interfacing. IEEE Trans. Neural Syst. Rehabil. Eng., 21, 554-566.

Delivopoulos, E., Chew, D.J., Minev, I.R., Fawcett, J.W. \& Lacour, S.P. (2012) Concurrent recordings of bladder afferents from multiple nerves using a microfabricated PDMS microchannel electrode array. Lab Chip, 12, 2540-2551

Fisher, L.E., Miller, M.E., Bailey, S.N., Davis, J.A., Anderson, J.S., Rhode, L., Tyler, D.J. \& Triolo, R.J. (2008) Standing after spinal cord injury with four-contact nerve-cuff electrodes for quadriceps stimulation. IEEE Trans. Neural Syst. Rehabil. Eng., 16, 473-478.

Fisher, L.E., Tyler, D.J., Anderson, J.S. \& Triolo, R.J. (2009) Chronic stability and selectivity of four-contact spiral nerve-cuff electrodes in stimulating the human femoral nerve. J. Neural Eng., 6, 046010.

Fitzgerald, J.J., Lacour, S.P., McMahon, S.B. \& Fawcett, J.W. (2008) Microchannels as axonal amplifiers. IEEE Trans. Biomed. Eng., 55, 11361146.

FitzGerald, J.J., Lacour, S.P., McMahon, S.B. \& Fawcett, J.W. (2009) Microchannel electrodes for recording and stimulation: in vitro evaluation. IEEE Trans. Biomed. Eng., 56, 1524-1534.

FitzGerald, J.J., Lago, N., Benmerah, S., Serra, J., Watling, C.P., Cameron, R.E., Tarte, E., Lacour, S.P., McMahon, S.B. \& Fawcett, J.W. (2012) A regenerative microchannel neural interface for recording from and stimulating peripheral axons in vivo. J. Neural Eng., 9, 016010.

Garde, K., Keefer, E., Botterman, B., Galvan, P. \& Romero, M.I. (2009) Early interfaced neural activity from chronic amputated nerves. Front. Neuroeng., 2, 5.

Gore, R.K., Choi, Y., Bellamkonda, R. \& English, A. (2015) Functional recordings from awake, behaving rodents through a microchannel based regenerative neural interface. J. Neural Eng., 12, 016017.

Hamilton, S.K., Hinkle, M.L., Nicolini, J., Rambo, L.N., Rexwinkle, A.M., Rose, S.J., Sabatier, M.J., Backus, D. \& English, A.W. (2011) Misdirection of regenerating axons and functional recovery following sciatic nerve injury in rats. J. Comp. Neurol., 519, 21-33.

Hassler, C., Boretius, T. \& Stieglitz, T. (2011) Polymers for neural implants. J. Polym. Sci., 49, 18-33.

Hess, A. \& Young, J.Z. (1952) The nodes of Ranvier. Proc. R. Soc. Lond. B Biol. Sci., 140, 301-320.

Hsu, J.-M., Rieth, L., Normann, R.A., Tathireddy, P. \& Solzbacher, F. (2009) Encapsulation of an integrated neural interface device with Parylene C. IEEE Trans. Biomed. Eng., 56, 23-29.

Kim, B.J., Kuo, J.T.W., Hara, S.A., Lee, C.D., Yu, L., Gutierrez, C.A., Hoang, T.Q., Pikov, V. \& Meng, E. (2013) 3D Parylene sheath neural probe for chronic recordings. J. Neural Eng., 10, 045002.

Lago, N., Ceballos, D., Rodríguez, F.J., Stieglitz, T. \& Navarro, X. (2005) Long term assessment of axonal regeneration through polyimide regenerative electrodes to interface the peripheral nerve. Biomaterials, 26, 2021-2031.
Lago, N., Udina, E., Ramachandran, A. \& Navarro, X. (2007) Neurobiological assessment of regenerative electrodes for bidirectional interfacing injured peripheral nerves. IEEE Trans. Biomed. Eng., 54, 1129-1137.

Li, W., Rodger, D.C., Meng, E., Weiland, J.D., Humayun, M.S. \& Tai, Y.C. (2006) Flexible Parylene Packaged Intraocular Coil for Retinal Prostheses. In 2006 International Conference on Microtechnologies in Medicine and Biology. IEEE, Okinawa, Japan, pp. 105-108.

Li, W., Rodger, D.C. \& Tai, Y.C. (2008) Implantable RF-coiled chip packaging. In 2008 IEEE 21st International Conference on Micro Electro Mechanical Systems. IEEE, Tucson, Arizona, pp. 108-111.

Li, W., Rodger, D.C., Meng, E., Weiland, J.D., Humayun, M.S. \& Tai, Y.C. (2010) Wafer-level parylene packaging with integrated RF electronics for wireless retinal prostheses. $J$. Microelectromech. S, 19, 735-742.

Minev, I.R., Chew, D.J., Delivopoulos, E., Fawcett, J.W. \& Lacour, S.P. (2012) High sensitivity recording of afferent nerve activity using ultracompliant microchannel electrodes: an acute in vivo validation. J. Neural Eng., 9, 026005

Navarro, X., Krueger, T.B., Lago, N., Micera, S., Stieglitz, T. \& Dario, P. (2005) A critical review of interfaces with the peripheral nervous system for the control of neuroprostheses and hybrid bionic systems. J. Peripher. Nerv. Syst., 10, 229-258.

Negredo, P., Castro, J., Lago, N., Navarro, X. \& Avendaño, C. (2004) Differential growth of axons from sensory and motor neurons through a regenerative electrode: a stereological, retrograde tracer, and functional study in the rat. Neuroscience, 128, 605-615.

Ortiz-Catalan, M., Hakansson, B. \& Branemark, R. (2014) An osseointegrated human-machine gateway for long-term sensory feedback and motor control of artificial limbs. Sci. Transl. Med., 6, 257re6.

Pang, C., Cham, J., Nenadic, Z., Musallam, S., Tai, Y.-C., Burdick, J. \& Andersen, R. (2005) A new multi-site probe array with monolithically integrated parylene flexible cable for neural prostheses. Annu. Int. Conf. IEEE Eng. Med. Biol. Soc., 7, 7114-7117.

Quiroga, R.Q., Nadasdy, Z. \& Ben-Shaul, Y. (2004) Unsupervised spike detection and sorting with wavelets and superparamagnetic clustering. Neural Comput., 16, 1661-1687.

Ramachandran, A., Schuettler, M., Lago, N., Doerge, T., Koch, K.P., Navarro, X., Hoffmann, K.-P. \& Stieglitz, T. (2006) Design, in vitro and in vivo assessment of a multi-channel sieve electrode with integrated multiplexer. J. Neural Eng., 3, 114-124.

Rodger, D.C., Weiland, J.D., Humayun, M.S. \& Tai, Y.-C. (2006) Scalable high lead-count parylene package for retinal prostheses. Sensor. Actuat. B Chem., 117, 107-114.

Rodger, D., Fong, A., Li, W., Ameri, H., Ahuja, A., Gutierrez, C., Lavrov, I., Zhong, H., Menon, P. \& Meng, E. (2008) Flexible parylene-based multielectrode array technology for high-density neural stimulation and recording. Sensor. Actuat. B-Chem., 132, 449-460.

Rutten, W.L.C. (2002) Selective electrical interfaces with the nervous system. Annu. Rev. Biomed. Eng., 4, 407-452.

Sabatier, M.J., To, B.N., Nicolini, J. \& English, A.W. (2011) Effect of axon misdirection on recovery of electromyographic activity and kinematics after peripheral nerve injury. Cells Tissues Organs, 193, 298-309.

Salzer, J.L. (1997) Clustering sodium channels at the node of Ranvier: close encounters of the axon-glia kind. Neuron, 18, 843-846.

Srinivasan, A., Guo, L. \& Bellamkonda, R.V. (2011) Regenerative microchannel electrode array for peripheral nerve interfacing. In $20115 \mathrm{th}$ International IEEE/EMBS Conference on Neural Engineering. IEEE, Cancun, Mexico, pp. 253-256.

Srinivasan, A., Tahilramani, M., Bentley, J.T., Gore, R.K., Millard, D.C. Mukhatyar, V.J., Joseph, A., Haque, A.S., Stanley, G.B., English, A.W. \& Bellamkonda, R.V. (2015) Microchannel-based regenerative scaffold for chronic peripheral nerve interfacing in amputees. Biomaterials, 41, 151-165.

Tansey, K.E., Seifert, J.L., Botterman, B., Delgado, M.R. \& Romero, M.I. (2011) Peripheral nerve repair through multi-luminal biosynthetic implants. Ann. Biomed. Eng., 39, 1815-1828.

Yao, L., de Ruiter, G.C.W., Wang, H., Knight, A.M., Spinner, R.J., Yaszemski, M.J., Windebank, A.J. \& Pandit, A. (2010) Controlling dispersion of axonal regeneration using a multichannel collagen nerve conduit. Biomaterials, 31, 5789-5797.

Yoo, P.B. \& Durand, D.M. (2005) Selective recording of the canine hypoglossal nerve using a multicontact flat interface nerve electrode. IEEE Trans. Biomed. Eng., 52, 1461-1469. 